\title{
Inclusión financiera de Colombia
}

\author{
Juan Carlos Urueña Mejía
}

Recibido: 19 de marco de 2015

Aprobado: 15 de mayo de 2015

Urueña, J. (2015). Inclusión financiera de Colombia. Activos, 24, 141-151.

\section{Clasificación JEL}

\section{G-21}

\section{Resumen}

En este trabajo, mediante la metodología exploratoria (revisión de fuentes secundarias), se busca mostrar cómo le ha ido a Colombia en términos de inclusión financiera [IF]. Para ello se describen algunas metodologías para medir la IF, como lo son el GLOBAL FINDEX, la AFI y la Asociación Latinoamericana de Instituciones Financieras para el Desarrollo [ALIDE]. Adicionalmente, se exponen las diferentes dimensiones de la IF, como lo son el acceso, el uso, la calidad y el bienestar que genera, y que en el caso de Colombia corresponde a que el 72,5 \% de la población tiene acceso a algún producto financiero.

\section{Palabras claves}

Inclusión financiera, ahorro, crédito.

1 Economista egresado de la Universidad Católica de Colombia. Magíster en Dirección y Administración de Empresas, de la Universidad Internacional de La Rioja, España. Docente de la Facultad de Ciencias Empresariales de la Corporación Universitaria Minuto de Dios. Correo electrónico: ujuancarlos@uniminuto.edu.co 
Urueña, J. (2015). Financial inclusion of Colombia. Activos, 24, 141-151.

\begin{abstract}
In this study using exploratory methodology (review of secondary sources) is intended to show how it has gone to Colombia in terms of Financial Inclusion (IF). To address this matter, some methodologies are described to measure IF such as the Global Findex, the AFI and ALIDE, further shows the different dimensions of the IF such as access, use, quality and wellness generated, in the case of Colombia, $72.5 \%$ of the population has access to any financial product.
\end{abstract}

\title{
Keywords
}

Financial Inclusion, saving, credits.

Urueña, J. (2015). Inclusion financière de la Colombie. Activos, 24, 141-151.

\section{Résumé}

Ce travail, veut montrer, à travers l'utilisation de la méthode exploratoire (révision de sources secondaires), comment va la Colombie en termes $\mathrm{d}$ 'inclusion financière (IF). Pour cela, 1 'on décrit quelques méthodes pour mesurer l'IF, tels que le GLOBAL FINDEX, la AFI et l'Association LatinoAméricaine des Institutions Financières pour le Développement (ALIDE). De plus, les différentes dimensions de l'IF y sont exposées, commel'accès, l'utilisation, la qualité et le bien-être qu'elle génère, et qui dans le cas de la Colombie correspond au fait que 72,5\% de la population a accès à au moins un produit financier.

\section{Mots clés}

Inclusion financière, épargne, crédit. 


\section{Introducción}

La inclusión financiera [IF] es un proceso que ha tomado una fuerza importante dentro de los mercados financieros a lo largo y ancho del mundo, en gran medida en países con sectores poblacionales de niveles de ingresos bajos o con una gran cantidad de ciudades o pueblos urbanos con poblaciones pequeñas y pueblos rurales, para lo cual ha sido importante acercar el sector financiero a dichos mercados y disminuir las brechas en el tema de desigualdad. Por tal motivo, en este artículo, mediante la metodología exploratoria, es decir, la revisión de fuentes secundarias, se busca mostrar cómo le ha ido a Colombia en términos de IF.

En los últimos años, a pesar de los destacados avances que se han hecho en términos de IF, todavía existen importantes rezagos y brechas por cerrar: niveles bajos de inclusión y bancarización financiera (ALIDE, 2014). En un estudio realizado por la Corporación Andina de Fomento (2011) en diferentes ciudades de América Latina ${ }^{2}$, se encontró que menos de la mitad de la población posee una cuenta bancaria; este estudio también reporta que tan solo un $24 \%$ de las personas adultas utiliza el sistema financiero para ahorrar, y el $19 \%$ para el manejo de créditos.

Partiendo de lo anterior, según Dueñas (2008), la IF se define como "el conjunto de instituciones que previamente autorizadas por el Estado están encargadas de captar, administrar e invertir el dinero tanto de personas naturales como de personas jurídicas (nacionales o extranjeras)" (p. 4).

De acuerdo con el Banco de la República, la IF corresponde al proceso en el cual se integran los servicios financieros a las actividades económicas diarias de las personas, y las cuales, a su vez, pueden contribuir de manera importante al crecimiento económico en la medida en que permitan disminuir de manera significativa los costos de financiación, aseguramiento

2 Ciudades de Brasil, Venezuela, Uruguay, Ecuador, Panamá, Colombia, Argentina, Perú y Bolivia. 
y manejo de los recursos para los agentes económicos tomadores de decisiones, es decir, las personas y las empresas (Banco de la República, 2014).

A su vez, el sistema financiero [SF] es un elemento fundamental de la IF, por tal razón, una de las funciones básicas del SF es captar excedentes de liquidez para transferirlas por medio de variados sistemas de financiación a unidades deficitarias (Dueñas, 2008), es aquí donde la IF empieza a revestir importancia.

\section{Metodologías para calcular la inclusión financiera}

Existen variados análisis y estadísticas en materia actual del estado de la inclusión financiera global, realizados por organizaciones, en los cuales se puede observar que no solo en Colombia, sino en distintos países se busca la IF (Bancoldex y Superintendencia Financiera de Colombia, 2013). Se tienen en cuenta tres organizaciones como las más relevantes por su fomento e impulso a la inclusión bancaria mundial.

En primer lugar, GLOBAL FINDEX es una base de datos que cuenta con información global de indicadores de IF. Se construyó con base en entrevistas realizadas a más de 150000 adultos de 148 países, en las que se tomaron medidas de cómo los adultos en dichas naciones podían ahorrar, pedir prestado, hacer pagos y gestionar el riesgo (Demirguc-Kunt \& Klapper, 2012).

En segundo lugar, la Alianza para la Inclusión Financiera [AFI], otra organización que plantea principios y guías comunes para sus miembros, también propone indicadores homogéneos para su medición y comparación entre países. Colombia es integrante de esta organización. La Banca de las Oportunidades, que tiene como objetivo promover el acceso al crédito y a los demás servicios financieros, muestra datos estadísticos del estado de la IF en Colombia (Bancoldex y Superintendencia Financiera de Colombia, 2013). 
Por último, la Asociación Latinoamericana de Instituciones Financieras para el Desarrollo [ALIDE] presenta diferentes factores influyentes ${ }^{3}$ en la IF de acuerdo con los estudios realizados entre sus miembros (ALIDE, 2014).

\section{Panorama de la inclusión financiera}

Según los estudios y las revisiones realizadas por ALIDE (2014), se refieren a tres términos que afectan la inclusión financiera actual:

La alfabetización financiera se refiere a poseer conocimientos, comportamientos, habilidades y actitudes necesarias para la toma de decisiones que le permitan determinar razonablemente qué opciones escoger en su beneficio; su ausencia afecta el ahorro (ALIDE, 2014).

La educación financiera se define como

el proceso a través del cual consumidores e inversionistas mejoran su entendimiento de los conceptos y productos financieros; y mediante la información, instrucción y orientación objetiva, desarrollan las habilidades y la confianza para ser más conscientes de los riesgos y las oportunidades financieras que les permitan tomar decisiones informadas, saber a dónde recurrir por ayuda y tomar otras medidas efectivas para mejorar su bienestar y seguridad. (ALIDE, 2014).

De acuerdo con ALIDE (2014), la capacidad financiera es "la habilidad y oportunidad de usar el conocimiento y las habilidades implicadas en la alfabetización financiera" o "es la combinación de conocimiento, habilidades y actitudes, con la oportunidad de aplicarlas". Es un concepto amplio que necesariamente vincula el funcionamiento individual a las entidades del sistema financiero (ALIDE, 2014).

3 Falta de ingresos suficientes, altos costos de transacción, excesiva documentación y trámites, desconocimientos y falta de confianza en el sistema financiero. 
GLOBAL FINDEX presenta cifras reveladoras sobre las personas que no tienen acceso a servicios bancarios. El $55 \%$ de quienes piden préstamos en las economías en desarrollo usa fuentes informales de crédito (préstamos a familiares y amigos). A nivel mundial, el $33 \%$ de los que tienen entre 15 y 24 años tiene menos probabilidades de tener una cuenta, y el $40 \%$ presenta menos probabilidades de haber ahorrado formalmente (Demirguc-Kunt \& Klapper, 2012).

En Colombia, por ejemplo, el 72,5 \% de la población tiene acceso a algún producto financiero (figura 1), principalmente cuentas de ahorro, sin embargo, la tercera parte de este porcentaje no lo ha utilizado en los últimos seis meses (Cano, 2014).

Figura 1. Evolución Indicador de Bancarización

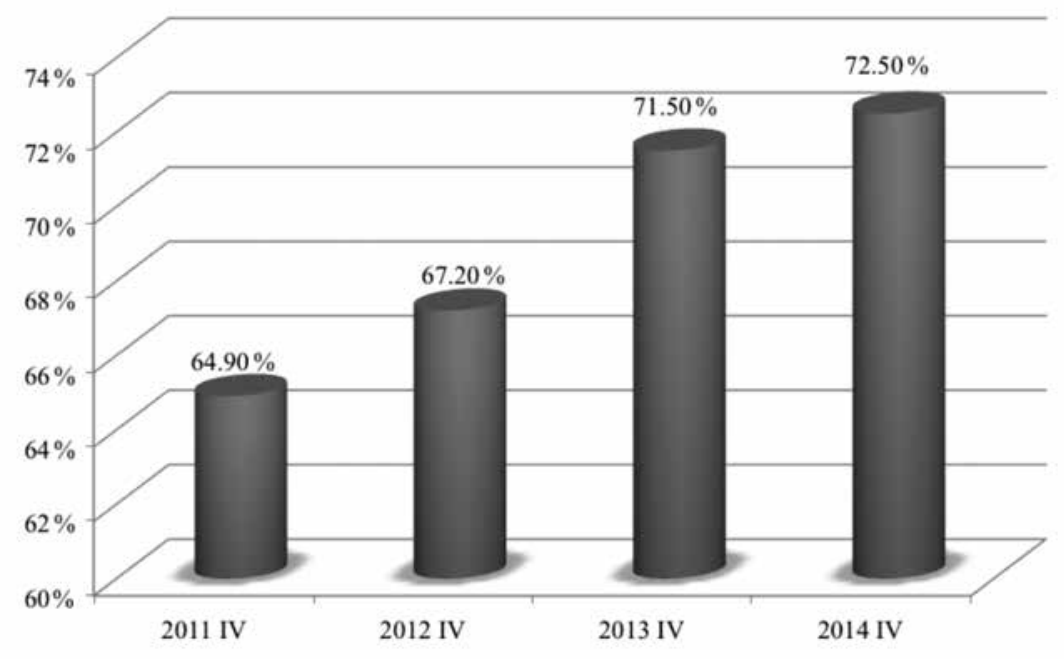

Fuente: CIFIN, DANE, ASOBANCARIA.

Los adultos con educación terciaria tienen más del doble de probabilidades de tener una cuenta formal que quienes tienen educación primaria o de menor nivel. El $35 \%$ de los residentes urbanos de bajos ingresos tiene una cuenta formal, mientras que el $22 \%$ corresponde a población rural. GLOBAL FINDEX expone que la banca móvil contribuye a reducir las brechas existentes en cuanto al ingreso financiero, ya que un tercio de adultos 
culpa al costo de apertura y al mantenimiento de una cuenta, o señala que los bancos están demasiado lejos, y esto permite reducirlos.

La Banca de Oportunidades, en conjunto con la Superintendencia Financiera de Colombia, en su reporte de 2012 expone los siguientes puntos medibles más importantes para conocer la situación de Colombia en cuanto a la IF.

Uso:

El porcentaje de la población adulta que cuenta con al menos un producto financiero, ya sea de ahorro y/o crédito, es del $67 \%$, lo que equivale a 20.8 millones de personas adultas bancarizadas, ya para 2014 se evidencia que este porcentaje de la población se ubica en un $72.5 \%$. En términos de crecimiento, para el periodo de análisis, el incremento de la población adulta bancarizada ha sido del $19 \%$, manteniendo un ritmo constante de crecimiento anual en los últimos años cercano al $5 \%$, porcentaje superior al crecimiento anual de la población adulta en Colombia, que muestra niveles de $2 \%$.

Figura 2. Evolución anual número de personas con cuenta de ahorros

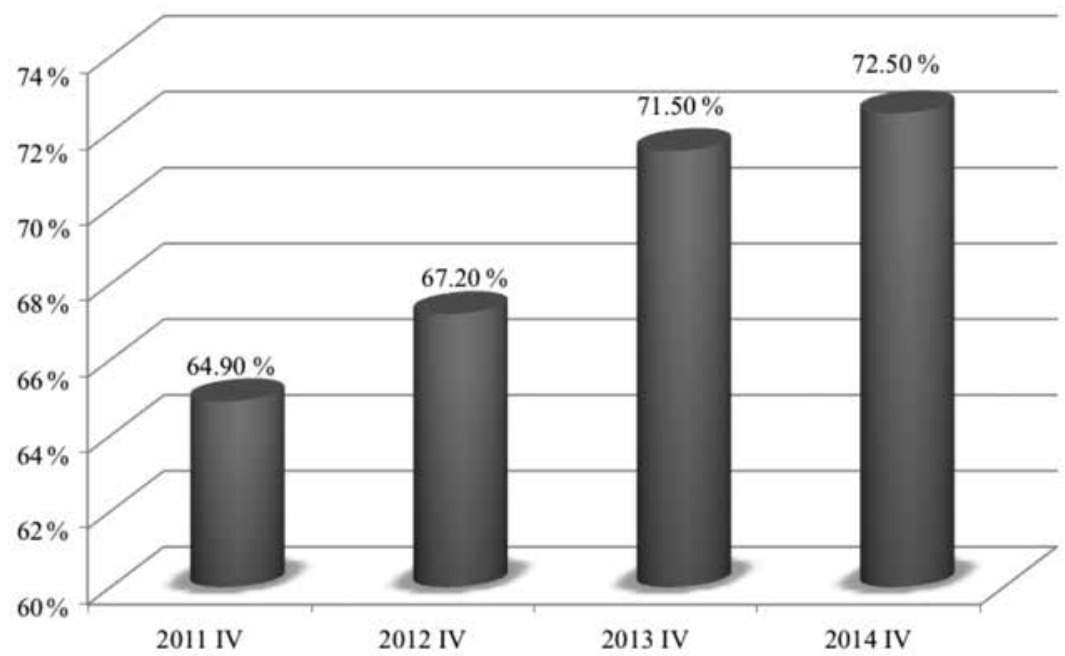

Fuente: CIFIN, DANE, ASOBANCARIA. 
Puntos de contacto:

Los canales por los cuales los habitantes pueden acceder a productos financieros y determinar cuál ha sido su crecimiento (Bancóldex y Superintendencia Financiera de Colombia, 2013).

\section{Calidad y bienestar, dimensiones de la inclusión financiera [IF]}

Luego de ver algunas metodologías para el análisis de su medición, se debe resaltar la importancia de la IF para Colombia. Algunos estudios dicen que existe una relación positiva entre la inclusión financiera y el crecimiento económico del país y, por consiguiente, en el bienestar de las personas (Superintendencia Financiera de Colombia, 2012). Varios trabajos de la literatura internacional han demostrado el vínculo entre el desarrollo del sector financiero y el crecimiento del PIB per cápita.

Basados en el planteamiento de la AFI, existen diferentes dimensiones para medir la inclusión financiera: la primera de ellas corresponde al uso, la segunda al acceso, la tercera es la calidad y la última es el bienestar (Bancóldex y Superintendencia Financiera de Colombia, 2013).

La primera evalúa la profundidad y permanencia en la utilización de los productos y servicios financieros; busca cuantificar la frecuencia y duración de la utilización, así como los portafolios de productos a disposición de las personas. La segunda evalúa la capacidad de los individuos para usar los productos y servicios ofrecidos por el sistema financiero. La tercera establece el tipo de productos y servicios que se ofrecen por el sistema financiero con las necesidades y percepción del consumidor; esta dimensión evalúa la calidad y profundidad de la relación entre las instituciones y el consumidor financiero, así como las alternativas disponibles y el grado de comprensión que el consumidor tiene de estas (Bancóldex y Superintendencia Financiera de Colombia, 2013). 
Figura 3. Dimensiones de la inclusión financiera

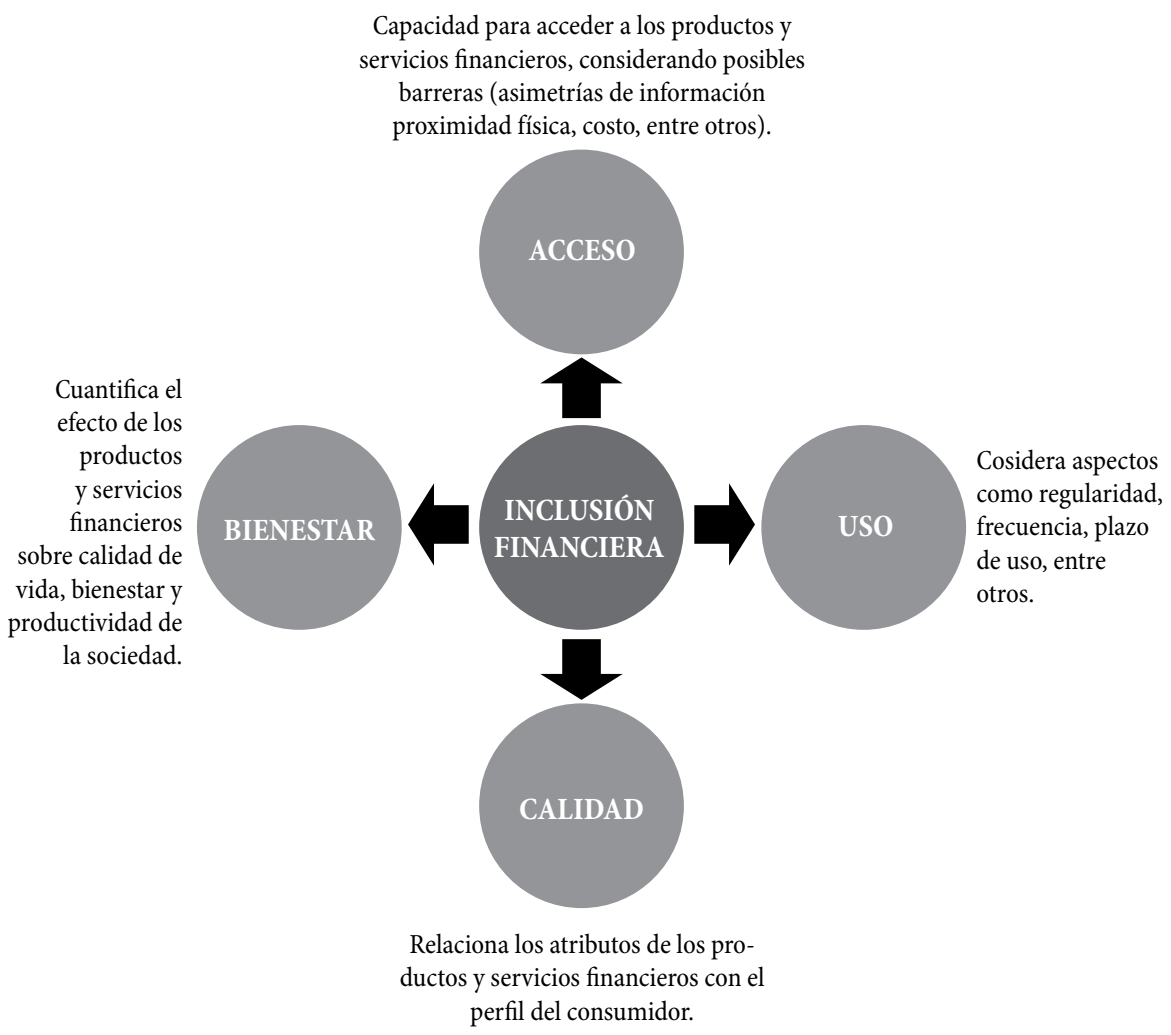

Fuente: tomado de Bancoldex \& Superintendencia Financiera de Colombia, 2013.

Respecto a la dimensión de bienestar, mide la influencia de un producto o servicio financiero particular sobre las condiciones de vida del consumidor financiero, la actividad empresarial que este realiza, sus niveles de productividad y, desde luego, el bienestar de los consumidores (figura 3).

Como se puede evidenciar, luego de realizar la revisión de algunas metodologías y ver el panorama, la IF es un tema que tiene auge en este momento en el país, ya que con él se puede lograr un mayor crecimiento económico y la ampliación de las oportunidades de inversión. 


\section{Conclusiones}

En los últimos años, el análisis y la promoción de la IF ha empezado a ser un tema central en las agendas de gobierno, así como de reguladores y supervisores financieros en diversos países, tanto desarrollados como en vía de desarrollo. Existe un consenso generalizado acerca de la relación positiva que se establece entre sistemas financieros más incluyentes y el nivel de desarrollo económico de los países y el bienestar de la población.

Teniendo en cuenta los indicadores y las estadísticas realizadas por dichas entidades tanto en Colombia como en los diferentes países en los cuales se están ejecutando los proyectos para la IF, es evidente que aún se encuentra el reto para los gobiernos y para las entidades financieras de conocer y comprender las necesidades de las personas, con el fin de diseñar estrategias que les permitan expandir el mercado financiero.

Basados en el planteamiento de la AFI, existen diferentes dimensiones para medir la inclusión financiera: la primera de ellas corresponde al uso, la segunda al acceso, la tercera es la calidad y otra más es el bienestar.

Desde la academia se realizan diferentes programas de educación financiera que les permiten a los estudiantes conocer más acerca de la IF con el fin de poder acceder después al sistema financiero.

\section{Referencias bibliográficas}

Asociación Latinoamericana de Instituciones Financieras para el Desarrollo (ALIDE). (2014). Innovación e inclusión financiera en la banca de desarrollo. Recuperado de http://www.alide.org/fn12_fin_rev4_inclusion.asp Asobancaria. (2013). Reporte trimestral de inclusión financiera. Diciembre de 2013.

Recuperado de http://www.asobancaria.com/portal/page/portal/Asobancaria/ publicaciones/economica_financiera/reporte_bancarizacion/2013/

Banco de la República. (2014). Informe especial de estabilidad financiera. Bogotá:

Autor. 
Bancóldex y Superintendencia Financiera de Colombia. (2013). Reporte de inclusión financiera 2012. Bogotá: Autor. Recuperado de http://goo.gl/hRtc6p

Cano, C. et al. (2014). Acceso a servicios financieros en Colombia. Borradores de Economía, 776.

Corporación Andina de Fomento. (2011). Servicios financieros para el desarrollo: promoviendo el acceso en América Latina. Bogotá: CAF.

Demirguc-Kunt, A., \& Klapper, L. (2012). Measuring Financial Inclusion. The Global Findex Database. U.S.A.: Banco Mundial.

Dueñas, R. (2008). Introducción al sistema financiero y bancario. Bogotá: Politécnico Grancolombiano.

Superintendencia Financiera de Colombia. (2012). Reporte de inclusión financiera. Bogotá: Autor. 